\title{
Crohn's Disease Associated Pyoderma Gangrenosum Treated with Adalimumab
}

\author{
Despoina Kakagia1, Eleni Efremidou², Nikolaos Lyratzopoulos², Alexandros Mitrakas², Michael Pitiakoudis², \\ George Kouklakis ${ }^{3}$ \\ ${ }^{1}$ Democritus University in Thrace Medical School, Plastic, Reconstructive Surgery, Alexandroupolis, Greece \\ ${ }^{2}$ Democritus University in Thrace Medical School, General Surgery, Alexandroupolis, Greece \\ ${ }^{3}$ Democritus University in Thrace Medical School, Endoscopy, Alexandroupolis, Greece
}

\begin{abstract}
Pyoderma gangrenosum is a known, though infrequent, ulcerative skin lesion that is often associated with systemic inflammatory and immunologic diseases. Objective: To present the efficacy of Adalimumab, a fully human Tumour Necrosis Factor- $\alpha$ antagonist, in the treatment of pyoderma gangrenosum associated with Crohn's disease. Methods: A young male patient with pyoderma gangrenosum of the shin as the first sign of clinically active Crohn's disease, who was treated with Adalimumab, is herein presented. Results: Almost complete healing of the lesion was achieved after 12 weeks of treatment. Conclusions: Successful treatment outcome suggests that Adalimumab is not only a therapeutic option for Crohn's disease but may also be a safe and effective therapy for Pyoderma gangrenosum associated with the disease. Pyoderma gangrenosum should be suspected in any cutaneous ulcerative lesion occurring in patients with Crohn's disease.
\end{abstract}

Key Words: Adalimumab, Crohn's disease, immunomodulating agents, pyoderma gangrenosum

Received: 04.08.2010

Accepted: 21.10 .2010

\section{Introduction}

Pyoderma gangrenosum (PG) is an uncommon inflammatory ulcerative skin lesion that in $50 \%$ of cases is associated with systemic diseases including inflammatory bowel disease (IBD), several forms of arthritis, leukemia and proleukemic states, and hepatic and immunologic diseases (1). PG has been associated with clinically active or inactive Crohn's disease (CD). The pathophysiology is not clear, but impairment of neutrophil chemotaxis has been suggested as the immunologic background (1).

Treatment options are topical and systemic. Corticosteroids and immuno-modulating agents are the mainstay treatment (2). Tumour necrosis factor $-\alpha$ (TNF- $\alpha$ ) is a well known proinflammatory mediator implicated in the pathogenesis of IBD. TNF- $\alpha$ antagonists have brought new perspectives to management of the extra-intestinal manifestations of this disorder, including PG (2).

The first anti-TNF- $\alpha$ agent that showed efficacy in luminal or fistulizing CD was infliximab (IFX) (3), a chimeric anti-TNF- $\alpha$ monoclonal antibody. Adalimumab is a fully human recombinant IgG1 monoclonal antibody that binds with high affinity and specificity to both soluble and membrane-bound TNF- $\alpha$ and has been recently introduced in the treatment of moderate to severe $C D$, and as therapy for maintenance of remission (4).

Adalimumab has been shown to be effective as induction therapy in anti-TNF- $\alpha$-naïve patients (4) as well as in patients with loss of response or intolerance to infliximab (IFX), as it lacks crossimmunogenicity with IFX (5). However, reports on the efficacy of adalimumab in CD associated PG are sparse.

A male patient with $P G$ of the shin as the first sign of active $C D$ who was successfully managed with Adalimumab is herein presented.

\section{Case Report}

A 34 -year-old male patient presented with a year's history of cutaneous red papules of the shin and sporadic diarrhoic episodes. As Figure 1 shows, the skin lesion progressed to ulceration after spontaneous rupture and possible minor injury.

Five months after the onset of the diarrhoic episodes, the patient was investigated by triplex of the lower limbs that was reported as normal and he was referred to the gastroenterologist for further investigation. Gastroscopy, coloscopy and biopsies established the diagnosis of CD. The patient was started on systemic methylprednisolone (starting dose 8 mg daily) for 3 months and Azathioprin (starting dose $75 \mathrm{mg}$ increased up to $100 \mathrm{mg}$ ) for 6 months. He also received topical treatment with corticosteroids for 4 months. As there was no response regarding both $C D$ and $P G$, he was referred to our clinic.

Blood test results on admission revealed iron deficiency anemia. Tuberculin Mantoux skin test, antinuclear antibodies, anti-PR3, anti-MPO, APCA, and AMA were negative. Thyroid 
function tests, $\lg E$ and $\lg M$ were found normal. chest radiograph, heart ultrasound audiogram and ophthalmologic examination of the patient revealed no abnormality.

Cultures obtained from the cutaneous lesion were all found negative for bacteria, fungi, mycobacteria and viruses. A skin biopsy revealed non specific infiltration by neutrophils, haemorrhage and epidermal necrosis, suggestive of pyoderma gangrenosum.

The patient was transfused and included in the adalimumab protocol, receiving an initial loading dose of $180 \mathrm{mg}$ followed by $80 \mathrm{mg}$ in 2 weeks and by $40 \mathrm{mg}$ every 2 weeks thereafter.

A clinical follow-up was performed every 15 days. Response to induction was assessed at 4 weeks from the initial dose of adalimumab. No side effects were recorded. The progress of healing of the cutaneous ulcer was monitored by digital photography and measurement of the wound surface was assessed by computerized planimetry (Texas Health Science Center at San Antonio Imagetool, version 3.0, as downloaded at http://ddsdx.uthsca.edu/dig/itdesc.html). Remission of CD and almost complete healing of PG was observed within 12 weeks of adalimumab therapy, as shown in Figure 2. The patient has remains asymptomatic for 13 months.

\section{Discussion}

The similarity in the bowel and skin in response to antiTNF- $\alpha$ factors may suggest common pathogenetic processes. However, the relationship between IBD and its extraintestinal manifestations remains unknown.

Pyoderma gangrenosum is an uncommon ulcerative skin lesion of uncertain etiology that can either be associated with systemic inflammatory diseases in at least $50 \%$ of cases or it can occur alone. Extraintestinal manifestations of IBD may occur in $21 \%$ to $36 \%$ of IBD cases and PG is one of them (6). The occurence of PD is irrelevant to the clinical activity of CD and sometimes, as in this case, it may herald active intestinal inflammation (7).

The classic ulcerative variant is usually observed on the legs, while a more superficial form known as atypical pyoderma gangrenosum tends to occur on the hands. Patients with pyoderma gangrenosum may have involvement of other organ systems that manifests as sterile neutrophilic abscesses such as the lungs, heart, central nervous system, gastrointestinal tract, eyes, liver, spleen, bones, and lymph nodes (1). The prognosis of pyoderma gangrenosum is generally good; however, recurrences may occur and residual scarring is not uncommon. Therapy may be topical or systemic, but prospective randomized controlled trials on any of the already applied treatments are lacking (8).

Cyclosporine was not used in our patient either systemically, due to nephrotoxicity, or topically by intralesional injections, as these are painful.

Surgery is not advised because of the pathergic phenomenon that may occur resulting in wound enlargement and even skin grafting has been reported to result in the development of pyoderma gangrenosum at the harvest site. Nevertheless, if surgery is necessary, the patient must be on therapy in order to prevent pathergy.
Anti-TNF- $\alpha$ agents, namely infliximab, etanercept and adalimumab, have proved to be effective treatment in Crohn's disease, rheumatoid arthritis, ankylosing spondylitis, ulcerative colitis, psoriasis and psoriatic arthritis (9). A growing number of published reports suggest that anti-TNF- $\alpha$ therapies may be effective in the treatment of numerous inflammatory skin diseases outside their currently approved indications such as pyoderma gangrenosum, sarcoidosis, dermatomyositis, scleroderma, hydradenitis suppuritiva, cicatricial pemphigoid, multicentric reticulohistiocytosis, Behçetıs disease, eosinophilic fasciitis, Sneddon-Wilkinson disease, pityriasis rubra pilaris, panniculitis and necrobiosis lipoidica diabeticorum (9).

Adalimumab has been shown in placebo-controlled clinical trials $(10)$ and uncontrolled studies $(4,5)$ to be effective as induction and maintenance therapy for inflammatory bowel disease. This fully human anti-TNF- $\alpha$ antibody is immunogenic (5), but the role that anti-adalimumab antibodies play in the loss of response and potential hypersensitivity reactions is unknown (8).

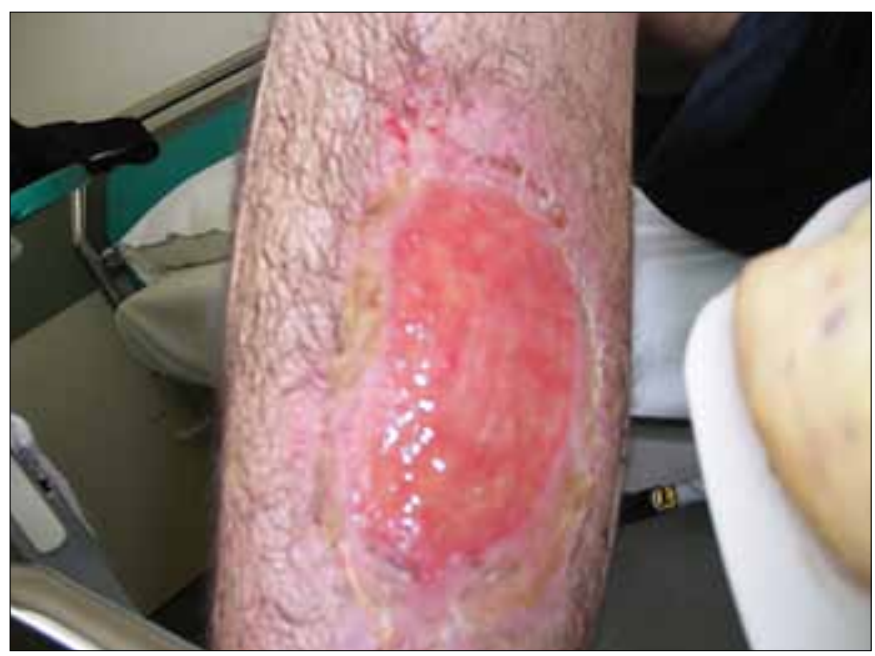

Figure 1. Skin ulceration expanding after rupture of red papules due to minor injury, combined with endoscopic findings of Crohn's disease, was suggestive of pyoderma gangrenosum

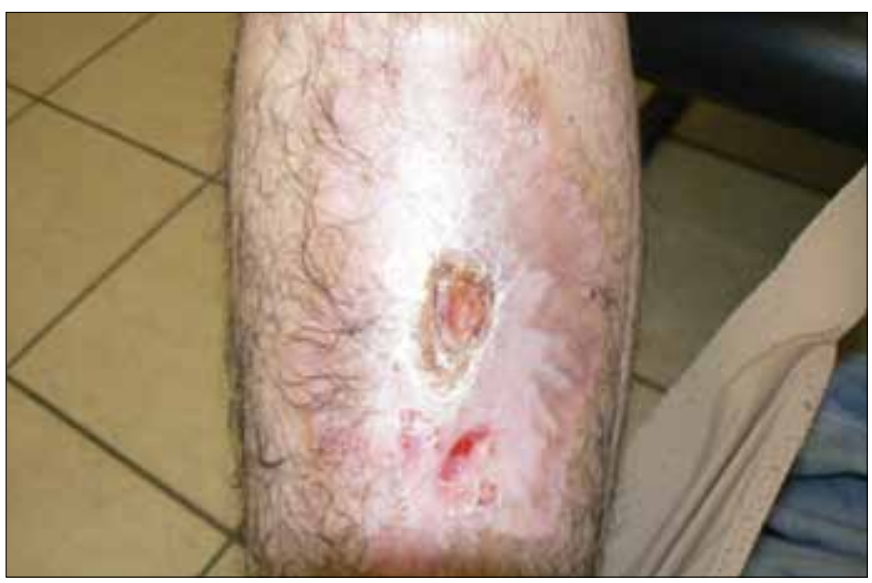

Figure 2. Almost complete healing of pyoderma gangrenosum after 12 weeks of treatment with adalimumab 
The patient herein presented was included in the Adalimumab protocol, as in our country adalimumab is approved even for anti-TNF- $\alpha$-naïve patients. Furthermore, the patient preferred it over infliximab, as he would be able to receive treatment at home. The safety of adalimumab in CD (5) is similar to that observed with other anti-TNF- $\alpha$ agents $(4,8)$. Most common side effects include inflammation at the injection site, rash, serious infections such as septic arthritis, cellulitis, pyelonephritis, diverticulitis and opportunistic infections. Also, the possible development of autoimmune diseases and malignancies have been reported $(5,10,11)$.

Adalimumab was effective in this patient with the ulcerative form of $P G$ and underlying $C D$. Further studies are essential to determine whether Adalimumab is effective in $P G$ associated with immunosuppression or inflammatory conditions or in exophytic and bullous forms of PG $(9,11)$.

\section{Conclusion}

Although adalimumab is a costly drug, it should be considered as alternative treatment for patients with PG and particularly those with corticosteroid-refractory or IFX resistant PG associated with IBD. Additional comparative studies are needed to determine the long-term efficacy and safety of antiTNF- $\alpha$ therapy and define its role in the management of PG, with or without accompanying IBD.

\section{Conflict of Interest}

No conflict of interest was declared by the authors.

\section{References}

1. Jacob SE, Weisman RS, Kerdel FA. Pyoderma gangrenosum-rebel without a cure? Int J Dermatol 2008;47:192-4. [CrossRef]
2. Barrie A, Regueiro M. Biologic therapy in the management of extraintestinal manifestations of inflammatory bowel disease. Inflamm Bowel Dis 2007;13:1424-9. [CrossRef]

3. Ljung $T$, Staun M, Grove O, Fausa O, Vatn MH, Hellstrom PM. Pyoderma gangrenosum associated with crohn disease: effect of TNF-alpha blockade with infliximab. Scand J Gastroenterol 2002;37:1108-10. [CrossRef]

4. Colombel JF, Sandborn WJ, Rutgeerts P. Adalimumab for maintenance of clinical response and remission in patients with Crohn's disease: the CHARM trial. Gastroenterology 2007;132:52-65. [CrossRef]

5. Hinojosa J, Gomollon F, García S. Efficacy and safety of short term adalimumab treatment in patients with active Crohn's disease who lost response or showed intolerance to infliximab: a prospective, open-label, multicentre trial. Aliment Pharmacol Ther 2007;25:409-18. [CrossRef]

6. Juillerat P, Mottet C, Froehlich F, Felley C, Vader JP, Burnand B, et al. Extraintestinal manifestations of Crohn's disease. Digestion 2005;71:31-6. [CrossRef]

7. Kouklakis G, Moschos J, Leontiadis GI, Kadis S, Mpoumponaris A, Molyvas E, et al. Infliximab for treatment of Pyoderma Gangenosum associated with clinically inactive Crohn's disease. A case report. Rom J Gastrenterol 2005;14:401-3.

8. Sands BE. Immunosuppressive drugs in ulcerative colitis: twisting facts to suit theories? Gut 2006;55:437-41. [CrossRef]

9. Alexis AF, Strober BE. Off-label dermatologic uses of anti-TNF-a therapies. J Cutan Med Surg 2005;9:296-302. [CrossRef]

10. Sandborn WJ, Hanauer SB, Rutgeerts P. Adalimumab for maintenance treatment of Crohn's disease: results of the CLASSIC II Trial. Gut 2007;56:1232-9. [CrossRef]

11. Stichenwirth M, Riedl E, Pehamberger H, Tappeiner G. Pyoderma gangrenosum in a patient with seronegative rheumatoid arthritis during therapy with adalimumab: toxic effects of adalimumab or failure of adalimumab to prevent the onset of this phenomenon? Arch Dermatol. 2008;144:817-8. [CrossRef] 